\title{
Cerebral Malaria: Insight into Pathology from Optical Coherence Tomography
}

\author{
Zhanhan Tu \\ University of Leicester \\ Jack Gormley \\ University of Liverpool \\ Viral Sheth \\ University of Leicester \\ Karl B. Seydel \\ Michigan State University \\ Terrie Taylor \\ Michigan State University \\ Nicholas Beare \\ University of Liverpool \\ Valentina Barrera \\ University of Liverpool \\ Frank A. Proudlock \\ University of Leicester \\ Chatonda Manda \\ University of Malawi \\ Simon Harding \\ University of Liverpool \\ Irene Gottlob ( $\nabla$ ig15@leicester.ac.uk ) \\ University of Leicester
}

\section{Research Article}

Keywords: Hand-held optical coherence tomography, cerebral malaria, malarial retinopathy, sequestration of parasitized red blood cells.

Posted Date: July 7th, 2021

DOI: https://doi.org/10.21203/rs.3.rs-117786/v2

License: @) (i) This work is licensed under a Creative Commons Attribution 4.0 International License. Read Full License

Version of Record: A version of this preprint was published at Scientific Reports on August 3rd, 2021. See the published version at https://doi.org/10.1038/s41598-021-94495-9. 


\section{Abstract}

\section{Purpose}

We aimed to investigate structural retinal changes in malarial retinopathy (MR) using hand-held optical coherence tomography $(\mathrm{HH}-\mathrm{OCT})$ to investigate its diagnostic potential.

\section{Methods}

Children with MR ( $\mathrm{n}=43$ ) underwent ophthalmoscopy, fluorescein angiography and HH-OCT during admission, one month $(n=31)$ and one year $(n=8)$ post-discharge. Controls were comatose patients without malaria $(n=6)$ and age/sex-matched healthy children $(n=43)$. OCT changes and retinal layer thicknesses were compared.

\section{Results}

On HH-OCT, hyper-reflective areas (HRAs) were seen in the inner retina of $81 \%$ of MR patients, corresponding to ischaemic retinal whiteningon fundus photography. Cotton wool spots were present in $37 \%$ and abnormal hyperreflective dots, co-localized to capillary plexus, in 93\%. Hyper-reflective vessel walls were present in $84 \%$, and intraretinal cysts in $9 \%$. Vascular changes and cysts resolved within 48 hours. HRAs developed into retinal thinning at one month $(p=0.027)$ whichwas more pronounced after one year $(p=0.009)$.

\section{Conclusions}

Ischaemic retinal whitening is located within inner retinal layers, distinguishing it from cotton wool spots. Vascular hyper-reflectivity may represent the sequestration of parasitized erythrocytes in vessels, a key CM feature. The mechanisms of post-ischemic retinal atrophy and cerebral atrophy with cognitive impairment may be similar in CM survivors. HH-OCT has potential for monitoring patients, treatment response and predicting neurological deficits.

\section{Summary}

Malarial retinopathy helps to diagnose cerebral malaria. Hand-held optical coherence tomography as an imaging biomarker, for the first time, used to visualize cerebral malaria pathophysiology, with potential for monitoring patient status and response to treatment.

\section{Introduction}

Cerebral malaria (CM) is a severe complication of Plasmodium falciparum infection. In 2017, approximately 219 million episodes resulted in death in $15-25 \%$ of patients and neurological morbidity in $30 \%$ of survivors. ${ }^{1-4}$ The pathogenesis is incompletely understood but intravascular sequestration of parasitized red blood cells (pRBCs) is a central feature. Sequestration occurs in various organs including the brain, leading to vascular congestion, 5,6 impaired perfusion, ${ }^{7}$, endothelial cell activation, ${ }^{8,9}$ blood-brain barrier breakdown ${ }^{10}$ and cerebral edema ${ }^{11}$, combined with systemic inflammatory responses ${ }^{12,13}$ and a prothrombotic state. ${ }^{14}$

Diagnosis of CM in endemic areas is difficult as asymptomatic parasitemia is common. On autopsy, $23 \%$ of children fulfilling WHO criteria for $\mathrm{CM}^{15}$ had non-malarial causes of death. ${ }^{5}$ 
Malarial retinopathy (MR) includes retinal whitening, white-centered hemorrhages and discolored vessels $2,5,16$ and is better than other clinical or laboratory features at distinguishing malarial from non-malarial coma. ${ }^{17}$ Severity of MR predicts prolonged coma, neurological sequelae and death. ${ }^{18}$ The exact time course of MR is unclear. ${ }^{13}$

Optical coherence tomography (OCT) allows non-invasive visualization and measurement of retinal layers at near microscopic resolution and has revolutionized diagnosis and treatment of retinal diseases. Recently, hand-held OCT (HH-OCT), suitable for children and comatose patients, has been developed. ${ }^{19} \mathrm{HH}-\mathrm{OCT}$ has the potentials to be a clinical tool in human CM.

Our purpose was to characterize retinal features of $\mathrm{CM}$ in vivo using $\mathrm{HH}-\mathrm{OCT}$ and to evaluate its potential to improve diagnosis and management. We aimed to: 1) characterize OCT changes in MR and compare them to fundus photography, fluorescein angiography (FA), and histological features seen in other CM patients in postmortem studies; 2) investigate the specificity of OCT features by comparing CM patients to patients in coma due to other causes and healthy controls; 3 ) compare the thickness of retinal layers in CM patients, control subjects, and clinical parameters; 4) describe longitudinal changes.

\section{Material And Methods}

\section{Study design and ethics}

This prospective observational study was performed at Queen Elizabeth Central Hospital (QECH) in Blantyre, Malawi (February 2016 to April 2017). The data collection protocol was approved by the ethical review committee of University of Malawi, College of Medicine and implemented in accordance with the ethical standards of the 1964 Declaration of Helsinki and its later amendments. As all the participants were younger than 12 years old, informed written consent was obtained from parents/guardians.

\section{Participants}

\section{Cerebral malaria}

All new cases with the diagnosis of CM admitted to the Pediatric Research Ward were approached. Inclusion criteria were: 1) Blantyre Coma Score $\leq 2$ (see Supplementary Table $S 1^{20}$ ); 2) peripheral parasitemia with $P$. falciparum;3) MR, and 4) no other discernible cause of coma ${ }^{1}$. MR was defined as: 1) retinal hemorrhages; 2) retinal whitening; and/or 3 ) orange or white retinal vessels seen on fundus examination. Isolated papilledema did not indicate MR. ${ }^{5}$

Standard clinical care and antimalarial treatment (intravenous artesunate) were instituted. ${ }^{20}$ In patients whose vital signs were stable, ophthalmoscopy, color photos (Topcon50-EX, Tokyo), FA ${ }^{21}$ were performed at admission. There were 43 children with $\mathrm{CM}$ included in this study. HH-OCT scans were repeated daily until discharge to detect the changes during the acute phase, and, in survivors, at one month and one year after discharge when possible.

\section{Control participants}

There were two groups of control participants recruited:

\section{1) Comatose children without $\mathrm{CM}$}

To exclude any OCT changes due to coma but not to $P$. falciparum infection, 6 comatose children without CM were included as a control group. According to WHO guidelines, inclusion criteria for comatose children without CM were 
children with Blantyre Coma Score $\leq 2$ but without peripheral parasitemia of $P$. falciparum. Parents/guardians of 6 comatose patients without parasitemia have been approached. They have all agreed to participate in the study.

2) Healthy children

Healthy local participants with normal visual acuity and fundi were recruited from other pediatric clinics/wards and local community around hospital, if parents/guardians had sufficient time to participate. They were age- and sexmatched to patients. Children with history of ophthalmological, neurological abnormalities or CM were excluded. Three out of the 46 healthy children were excluded due to poor cooperation.

\section{Optical Coherence Tomography (HH-OCT)}

Macula HH-OCT scans (Leica Microsystems ${ }^{\mathrm{TM}}$ Envisu C-Class, Milton Keynes, UK) of 12 x 8 x $2 \mathrm{~mm}$ volume were obtained (600 A scans and 80 B scans) (see Supplementary Figure S1, example of a control child). Averaging scans is not possible with the hand-held OCT as small movements of the probe or patients will cause artefacts. At admission, pathological changes on HHOCT scans collected from CM patients were compared to fundus pictures (see Supplementary Figure S1D, example of a control child) and FAs (see Supplementary Figure S1E, example of a control child) taken from the same children. For comparison of the same retinal areas location of retinal vessels was used for the three different image modalities (for methodology see Supplementary Figure S1, example of a control child). HH-OCT scans from CM patients were also compared with scans collected from two control groups.

Thickness of inner and outer retina was measured with manual segmentation (custom macro;

http://imagej.nih.gov/ij/; National Institutes of Health, Bethesda, USA) at the foveal center and parafovea between 4 to $6^{\circ}$ nasally and temporally (see Supplementary Figure S1C). ${ }^{22}$ Blurry OCT images where retinal layers could not be clearly identified were excluded and retaken until images with sufficient quality were obtained.

\section{Statistical Methods}

Linear mixed models (SPSS, version 24, IBM Analytics) adjusted for eye (right/left eye), gender and age were used to investigate: 1) differences in retinal layer thicknesses between patients and controls; 2) longitudinal changes in thicknesses of retinal layers; 3) correlation with clinical data. Bonferroni post-hoc correction was applied for multiple comparisons.

\section{Results}

\section{Characteristics of subjects}

Demographic and clinical characteristics of CM patients and healthy controls are shown in Table 1 and Table 2, respectively. On admission, OCT scans were collected from 43 patients with CM. Sixty-eight high quality scans were utilized for quantitative measurements. Forty-three healthy controls were recruited, and 68 OCT images were analyzed to match ages of the CM patients from whom good quality images were obtained. There were 31 OCT images analyzed at 1-month post discharge and 15 OCT images at 1-year post discharge from CM patients and controls. Six comatose children without CM (12 eyes) were recruited (see Supplementary Table S2). Visual acuities were within normal age range for all patients at discharge and at follow-up examination and for all healthy control children (0.25Ds to $+0.50 \mathrm{DS}) .{ }^{23}$ None of the participants had cataract and floaters during indirect fundus examinations. We have also checked en-face OCT scans, and eliminated OCT scans with artifacts from analysis. 
Table 1

Demographic data of patients with cerebral malaria (CM) and controls on admission and follow-up visits

Cerebral Malaria Patients on Admission

\begin{tabular}{|c|c|c|c|c|c|c|c|c|}
\hline \multirow[t]{2}{*}{ Groups } & \multirow{2}{*}{$\begin{array}{l}\text { Number of } \\
\text { participants }\end{array}$} & \multicolumn{2}{|c|}{ Age (months) } & \multirow{2}{*}{$\begin{array}{l}\text { Number } \\
\text { of } \\
\text { males }\end{array}$} & \multicolumn{4}{|c|}{ OCT images (eyes) } \\
\hline & & Range & Mean & & $\begin{array}{l}\text { On } \\
\text { admission }\end{array}$ & $\begin{array}{l}24 \text { hours } \\
\text { after } \\
\text { admission }\end{array}$ & $\begin{array}{l}48 \text { hours } \\
\text { after } \\
\text { admission }\end{array}$ & $\begin{array}{l}\text { Fluorescein } \\
\text { angiography } \\
\text { (eyes) }\end{array}$ \\
\hline $\begin{array}{l}\text { CM } \\
\text { patients }\end{array}$ & 43 & $\begin{array}{l}7- \\
131\end{array}$ & 55 & 21 & $\begin{array}{l}86 \text { (68 } \\
\text { high } \\
\text { quality) }\end{array}$ & 84 & 48 & 64 \\
\hline $\begin{array}{l}\text { Healthy } \\
\text { Controls }\end{array}$ & 43 & $\begin{array}{l}9- \\
138\end{array}$ & 56 & 21 & $\begin{array}{l}68 \\
\text { analyzed }\end{array}$ &.. &.. &.. \\
\hline
\end{tabular}

Cerebral Malaria Patients at 1 Month Follow-up

$\begin{array}{lll}\text { Groups } & \text { Age (months) } & \text { OCT images (eyes) }\end{array}$

\begin{tabular}{|c|c|c|c|c|c|c|}
\hline $\begin{array}{l}\text { Number of } \\
\text { patients }\end{array}$ & Range & Mean & $\begin{array}{l}\text { Number } \\
\text { of } \\
\text { males }\end{array}$ & $\begin{array}{l}\text { Total } \\
\text { scans }\end{array}$ & $\begin{array}{l}\text { With } \\
\text { HRAs }\end{array}$ & $\begin{array}{l}\text { Without } \\
\text { HRAs }\end{array}$ \\
\hline
\end{tabular}

$\begin{array}{llllllll}\begin{array}{l}\text { CM } \\ \text { patients }\end{array} & 18 & \begin{array}{l}12- \\ 96\end{array} & 54 & 8 & \begin{array}{l}36(31 \\ \text { high } \\ \text { quality })\end{array} & 18 & 13 \\ & & & & \end{array}$

$\begin{array}{llllll}\text { Healthy } & 18 & 11- & 55 & 8 & 31 \\ \text { Controls } & & 107 & & & \text { analyzed }\end{array}$

Cerebral Malaria Patients at 1 Year Follow-up

\begin{tabular}{|c|c|c|c|c|c|c|c|c|}
\hline \multirow[t]{2}{*}{ Groups } & \multicolumn{4}{|c|}{ Age (months) } & \multicolumn{2}{|c|}{ OCT images (eyes) } & \multirow[b]{2}{*}{$\begin{array}{l}\text { Without } \\
\text { HRAs }\end{array}$} & \multirow[b]{2}{*}{.. } \\
\hline & $\begin{array}{l}\text { Number of } \\
\text { patients }\end{array}$ & Range & Mean & $\begin{array}{l}\text { Number } \\
\text { of } \\
\text { males }\end{array}$ & $\begin{array}{l}\text { Total } \\
\text { scans }\end{array}$ & $\begin{array}{l}\text { With } \\
\text { HRAs }\end{array}$ & & \\
\hline $\begin{array}{l}\text { CM } \\
\text { patients }\end{array}$ & 8 & $\begin{array}{l}26- \\
140\end{array}$ & 73 & 4 & $\begin{array}{l}16(15 \\
\text { high } \\
\text { quality) }\end{array}$ & 13 & 2 & .. \\
\hline $\begin{array}{l}\text { Healthy } \\
\text { Controls }\end{array}$ & 8 & $\begin{array}{l}26- \\
140\end{array}$ & 73 & 4 & $\begin{array}{l}15 \\
\text { analyzed }\end{array}$ &.. &.. & .. \\
\hline
\end{tabular}

\section{OCT findings in children with MR on admission}

\section{Hyper-reflective capillaries}

Abnormal hyper-reflective well-defined dots located in the parafoveal region were seen on OCT scans in $93 \%$ of CM eyes (Fig. 1.1.A) in the area corresponding to capillary beds of the superficial, intermediate and deep retinal plexuses (green, blue and orange arrows respectively) (Fig. 1.1.A and $\mathrm{E}^{24}$ ). Histology of MR from a different patient ${ }^{25}$ not included in the OCT study (Fig. 1.1.D) shows parasite sequestration in capillaries and venules (black 
arrows - high sequestration vs. black arrowheads - low sequestration) in similar locations as hyper-reflective dots. Affected capillaries were not visible on en-face OCT (Fig. 1.1.B) and color photograph (Fig. 1.1.C) and occurred with corresponding capillary non-perfusion seen on FA (Fig. 1.1.F). To show the differences between the abnormal hyperreflective vessels and normal vessels in a healthy control on a B-scan, a side-by-side comparisons is shown in Supplementary Figure S2.A (CM patient) and S2.B (healthy control).

\section{Hyper-reflective vessels}

Hyper-reflective ring-like or ovoid structures (Fig. 1.2.A and B) corresponding to the location of retinal vessels on enface images (Fig. 1.2.C), were seen on OCT in $90 \%$ of eyes. The walls of the veins, arteries, venules and arterioles were hyper-reflective on OCT (Fig. 1.2.A and 1.2.B). The lumina were hyper-reflective (84\%) (Fig. 1.2.A; red arrow) or hypo-reflective (73\%) (Fig. 1.2.B; yellow arrow). In 67\% of eyes both were present simultaneously. Figure 1.2.D (red arrow) represents histology from a different patient not included in the OCT study shows a vessel where both wall and lumen are filled with parasitized red blood cells (pRBCs), comparable to vessels on OCT with hyper-reflective lumina (example in Fig. 1.2.A (red arrow)). Figure 1.2.D (yellow arrow) shows dense sequestration of erythrocytes on the vessel wall with preservation of the blood flow in the hypo-reflective lumen comparable to OCT with hyporeflective lumen (Fig. 1.2.B yellow arrow). Hyper-reflective vessels looked predominantly normal on FA (Fig. 1.2.E) and funduscopy (Fig. 1.2.F). Side-by-side comparisons of vascular walls of a b-scan of a normal healthy age-and gender-matched eye (see Supplementary FigureS2.D) is shown for comparison with hyper-reflective vessels in patients with CM (see Supplementary Figure S2.C).

Hyper-reflective areas (HRAs)

Hyper-reflective areas (HRAs) with variable size and diffuse borders, appeared as high reflectance in the inner nuclear layer (INL), outer plexiform layer (OPL) and the outer nuclear layer (ONL) (Fig. 2.1.A; white circle and B, white arrows) in $81 \%$ of eyes. Larger HRAs corresponded to dark areas on en-face image (Fig. 2.1.C; white arrows). They were located predominantly in the parafoveal area with a propensity for the temporal raphe (watershed zone) and spared the foveolae. Histology from the literature ${ }^{17}$

shows immunochemical staining of fibrinogen leaking from vessels into the OPL (Fig. 2.1.D black arrowhead) suggestive of breakdown of the blood retinal barrier at similar locations and distributions than HRAs. Larger HRAs were visible as capillary non-perfusion on FA (Fig. 2.1.E; white arrows), and whitening on fundus photos (Fig. 2.1.F; white arrows) while small HRAs were not always apparent ${ }^{26}$.

\section{Cotton wool spots (CWS) (Fig. 2.2)}

CWS were distinct from HRAs on OCT, being located in the retinal nerve fiber layer (RNFL), raised and well circumscribed. CWS distorted inner and outer retinal layers with decreased signal density compared to HRAs (Fig. 2.2.A) (37\% of eyes). They were less straightforward to distinguish from retinal whitening on color photos as white/grey feathered spots (Fig. 2.2.E). ${ }^{26}$ On en-face OCT, CWS had a dark appearance (Fig. 2.2.B). Histology from a different patient ${ }^{17}$ not included in the OCT study (Fig. 2.2.C) shows b-APP staining in the RNFL indicative of axonal damage located in the same retinal layers as CWS. On FA CWS corresponded to grey areas masking underlying capillaries (Fig. 2.2.D).

\section{White-centered hemorrhage (see Supplementary Figure S3.1)}

White-centered hemorrhages corresponded to superficial well-circumscribed lesions below the ILM, or to deeper diffuse hyper-reflective lesions involving the inner retina. En-face OCT images show superficial hemorrhages as 
dark spots or rings matching hemorrhages on color images. Deep white-centered hemorrhages are poorly defined on en-face imaging.

\section{Cystoid macular edema (CME) (see Supplementary Figure S3.2)}

CME was present on OCT in four patients (9\%) with and without sub-retinal fluid. Cysts were in the OPL of the foveal and parafoveal region: none was detected fundoscopically in any patients.

The frequencies of the various OCT characteristics in patients with MR are summarized in Supplementary Table S3.

\section{OCT findings in control children}

None of the healthy or comatose control children had any retinal abnormalities on OCT.

\section{Quantitative Analysis}

\section{Macular and foveal thickness}

On admission, no difference in retinal thickness at the foveal center and parafovea between 4 to $6^{\circ}$ nasally and temporally was found between CM patients and controls, although there was a trend to statistical significance for thicker inner retinal layers temporally in CM group $(p=0.06)$.

Coma resolution time was positively correlated with the thickness of outer retinal layers of temporal macula $(p=$ $0.0036)$ on admission. Thicker outer retinal layers were found in children with longer coma resolution time. No correlations between thickness of retinal layers and other clinical parameters were found.

\section{Longitudinal findings}

\section{Vascular changes}

Twenty-four hours after starting treatment, the intensity of the hyper-reflective capillaries and vessels diminished on OCT, returning to normal by 48 hours in all patients (Fig. 3).

\section{Hyper-reflective areas (HRAs) and retinal atrophy}

HRAs were still visible one-month post-discharge in 18 eyes (Fig. 3C). Seven out of eight patients who had one-year follow-up had HRAs at admission, of which five showed visible retinal atrophy corresponding to the previous location of HRAs (Fig. 3D). Most of the volume loss occurred in the inner retina temporally, consistent with the anatomical location of HRAs. The patient without HRAs had no noticeable retinal thinning at one-year.

\section{Longitudinal quantitative retinal thickness}

\section{Comparison of retinal layer thickness between admission and follow-up in CM}

Compared to admission, significant thinning of inner retinal layers $(p<0.0001)$ was observed at one-month for the entire CM cohort (31 eyes; and patients; 18 eyes with and 13 eyes with and without HRAs) (see Supplementary Figure S4.A). No differences in the outer retinal thickness were observed, except at the foveola where a significant increase of the outer retinal layers was observed $(p<0.001)$. At one-year follow-up (15 eyes), the thickness of the 
inner layers had significantly decreased at the temporal parafovea compared to admission $(p<0.001)$. (see Supplementary Figure S4.B)

As retinal atrophy was observed in locations corresponding to HRAs, CM patients with and without HRAs were compared. Significant thinning was found at one-month $(p=0.024)$ and one-year $(p=0.0009)$ in patients with HRAs on admission, but not in patients who did not have evidence of HRAs on admission (see Supplementary Figure S4). As only two eyes without HRAs were scanned at one-year, statistical analysis was not performed.

\section{Comparison of retinal layers thickness between healthy controls and CM patients at follow-up}

At one-month, no difference was found in the thickness of retinal layers between CM patients and controls.

However, when analyzed separately, patients with HRAs at admission $(n=18)$ had thinning in the inner layers at one month, especially on the parafoveal temporal side $(p=0.027)$, while no significant change was found in those without HRAs $(n=13)$ at admission (Fig. 4A).

At one-year the CM patients had significant temporal parafoveal thinning compared to controls $(p=0.024)(13$ eyes with HRAs at admission, $p=0.009$ ) (Fig. 4B).

No significant difference was found in outer retinal layers or RNFLs at follow-up.

\section{Table 2 Clinical data in CM patients}


Age (months)

Male sex - no. (\%)

Variable before admission

Duration of fever (hour)

Duration of coma (hour)

\section{Variable on admission}

Temperature $\left({ }^{\circ} \mathrm{C}\right)$

Blantyre coma score (\%)

0

1

2

Packed Cell Volume

CSF opening pressure ( $\mathrm{mm}$ of water)

Median

Interquartile range

Parasitemia (parasites $/ \mathrm{mm}^{3}$ )

Median

Interquartile range

HRP2 (ng/ml)

Median

Interquartile range

White cells $\left(\times 10^{-9} /\right.$ litre $)$

Median

Interquartile range

Platelets $\left(\times 10^{-9} /\right.$ litre $)$

Median

Interquartile range

Hypoglycemia - no. (\%)

Hyperlactatemia - no. (\%)

Discharge outcomes

Coma resolution time (hour)
$54 \pm 30$, Age range 7-131

21 (49)

$56 \pm 32$

$13 \pm 12$

$39 \pm 1$

2

13

18

$23 \pm 5 \cdot 9$

170

$115-240$

4697

$189-53,520$

1082

562 - 3177

8

$6 \cdot 4-11 \cdot 45$

64

$43 \cdot 3-88$

$0(0)$

15 (35)

$36 \pm 28$ 
Data with ' $\S$ ' presented as mean \pm SD. HRP2: histidine-rich protein 2 concentration; CSF: cerebrospinal fluid opening pressure; Hyperlactatemia: an increased level of lactate in the blood (> $5 \mathrm{mmol} /$ liter); Hypoglycaemia: the glucose (blood sugar) level lower than $3 \mathrm{mmol} /$ liter; Coma resolution time: the time from admission to recovery reaching and sustaining coma score 5.

\section{Discussion}

\section{Hyper-reflective capillaries and vessels}

Hyper-reflective rings or ovoid structures seen in the OCTs of CM patients clearly correspond to retinal vessels, and it is probable that the hyper-reflective walls represent endothelial sequestration of pRBCs, the histological hallmark of CM.

Small hyper-reflective dots are co-localized with retinal capillary plexus, strongly suggesting that they correspond to retinal capillaries affected by sequestration. Our hypothesis is that the hyper-reflectivity may be directly due to the presence of sequestered pRBCs or to a change exerted on the vessel wall. Their rapid resolution with treatment suggests a reversibility which mirrors parasite clearance.

A recent investigation of malarial retinopathy used a mouse model of cerebral malaria to compare changes in OCT to fundus imaging with labelled parasites and histology with immunological markers. ${ }^{27}$ It localized the parasites in larger vessels by detecting labelled GFP and in three capillaries layers with CF8 staining, confirming OCT hyperreflectivity in vessels with sequestration and supporting our conclusions. The locations and morphology of the hyper-reflectivity is consistent with our findings localizing the parasites by detecting GFP expression of clusters of plasmodia in big vessels and all three capillary layers with CF8 staining. ${ }^{27}$

Vessels with hyper-reflective lumens on OCT likely represent lumens filled with pRBCs, as uninfected RBCs have a low OCT signal. A hypo-reflective center in a hyper-reflective lumen is probably a narrow area of central patency. These findings are consistent with the retinal histology in CM. ${ }^{16}$ Hyper-reflectivity seen in retinal capillaries and vessels is most likely due to hemozoin, a metabolic by-product of the falciparum parasite. ${ }^{28}$ Hemozoin is birefringent on examination with polarised light. ${ }^{28}$ Retinal intravascular material seen in retinal vessels on FA indicates sequestration, and is present in the majority of MR positive patients (post-capillary $98.3 \%$ and small venules $87.9 \%$ ). ${ }^{16}$ This is very similar to our OCT data (93\% hyper-reflective capillaries and $90 \%$ hyper-reflective vessels) further supporting sequestration as the basis of the OCT findings. Vascular changes in the macula on OCT were rarely seen on funduscopy, likely representing the higher resolution and sensitivity of OCT. Vessel abnormalities are more visible in the peripheral retina on clinical examination.

Small hyper-reflective foci have also been found in other inflammatory retinal diseases such as diabetic retinopathy. ${ }^{29}$ However, these foci are also located in the outer retinal layers unlike the hyper-reflective dots we found in CM (see Supplementary Figure S5). Therefore, the hyper-reflective foci in diabetic retinopathy do not follow the distribution of vessels in contrast of the hyper-reflective dots in CM. 
Typically, after 48 hours of antimalarial treatment, retinal vessels and capillaries appeared normal on OCT. This time course was similar to peripheral blood clearance times (Table 2). Therefore, OCT has potential to noninvasively assess response to treatment and track the reversal of sequestration in a physiologically relevant setting, a CNS microvascular bed.

\section{Retinal whitening and hyper-reflective areas}

Retinal whitening in MR corresponds to capillary non-perfusion with good evidence it is caused by ischemia 16 which, at a cellular level may represent cytotoxic edema or oncotic cell swelling due to reduced capillary perfusion, ${ }^{30}$ metabolic steal by sequestered parasites ${ }^{22}$ or microthrombi. ${ }^{17}$ Immunochemical staining of fibrinogen leaking from vessels suggests that blood retinal barrier breakdown occurs at similar locations and distributions as HRAs. ${ }^{17}$ The co-location of larger HRAs to retinal whitening strongly suggests HRA's are a manifestation of tissue hypoxia.

Our group has previously shown association between severe retinal whitening with reduced electroretinography bwave amplitudes, indicating reduced function of the inner nuclear layer. This is consistent with involvement of the INL in HRAs. ${ }^{31}$ CWS are a recognized feature of MR, but can be difficult to differentiate from retinal whitening clinically and on photographs. OCT images are able to differentiate these retinal features more readily. ${ }^{21}$ Retinal whitening, mainly located parafoveally, appears to correspond to HRAs at the interface of the INL, OPL, and ONL; by contrast, CWS, located predominantly in the peripheral macula corresponded to hyper-reflective RNFL lesions.

Similar hyper-reflective lesions co-localized with retinal whitening have been described in paracentral acute middle maculopathy (PAMM), an uncommon condition seen in patients with retinal ischemia due to various aetiologies (See Supplementary Figure S6). ${ }^{32,33}$ As in MR the foveola is not affected as it is avascular and mainly supplied by choroidal blood flow. HRAs in PAMM and MR appear to be a feature of ischemia. ${ }^{34} \mathrm{CWS}$ are also whitish lesions, but with OCT are readily distinguishable from retinal whitening. CWS occur due to the disruption of axonal transport in the RNFL. In MR, CWS tend to occur in the peripheral macula and just outside the vascular arcades, and likely represent a border between ischemic and better perfused retina. ${ }^{35}$

\section{White centered hemorrhage and cystoid macula edema (CME)}

On OCT hemorrhages were hyper-reflective and either superficial and well-circumscribed, or deep and diffuse involving the inner retina likely reflecting hemorrhages from superficial and deep capillary plexus. White-centered hemorrhages are typical in MR and are associated histologically with fibrin thrombi (See Supplementary Figure S3.1.C). Central thrombi may be just discernible in deep hemorrhages on OCT.

CME is difficult to visualize and diagnose using ophthalmoscopy and has probably been missed in studies relying on ophthalmoscopy. It is easily visible on OCT and we found it in nearly $10 \%$ of patients (See Supplementary Figure S3.2.A). CME occurred less frequent in our patients, than in autopsy cases (50\%) ${ }^{17}$ which may be explained by the increased severity of disease on cases going on to autopsy. Larger numbers are needed to test whether CME is associated with fatal outcome.

\section{Atrophy of inner retinal layers}

Inner retinal layers started to thin at one-month follow-up and by one-year were clearly atrophic compared to admission, and to controls. Atrophy was associated with the presence of HRAs on admission, indicating the neuronal consequences of acute patchy ischemia on the CNS, and providing a mechanism for the cognitive 
impairment associated with brain atrophy in CM survivors. ${ }^{4}$ Comparison between retinal atrophy on OCT and brain atrophy on MRI as well as cognitive functions are necessary to elucidate to which extent they are associated.

\section{Correlation of size of retinal layers with clinical parameters}

Thicker outer layers were associated with longer coma resolution time, possibly indicating that edema of temporal outer retinal layers is associated with disease severity.

\section{Limitations of the study}

This is a novel study of new HH-OCT technology. Further work will be required to establish the role of HH-OCT from the promising potential we have identified, particularly with regard to diagnosis of $\mathrm{CM}$, tracking sequestration, and predicting cognitive sequelae.

OCT mainly images the macula within the vascular arcades, while MR changes occur throughout the fundus. However, most of MR features occur within the macula, and the higher resolution of OCT and ability to crosssection the retina enables it to detect previously undetected retinal effects.

We only had a small sample size for the one-year follow-up, but findings were statistically significant. We also had a small group with coma of non-malarial causes $(n=6)$. However, none of the larger group of healthy children had any of the MR signs seen on OCT.

Our interpretation of the pathological findings on OCT can only be to some extend speculative. This is because a direct comparison of the same cut of an OCT images and postmortem histology is impossible. It is difficult to obtain post-mortem pathology from the same patients from which we obtained OCT as the minority of patients are dying. In addition, retinal pathology changes over time with the course of the disease. For example, twenty-four hours after starting treatment, the intensity of the hyper-reflective capillaries and vessels diminished significantly on $\mathrm{OCT}$, returning to normal by 48 hours in all patients (see longitudinal changes in result section, Fig. 3). Therefore, even if we would obtain an eye of a deceased patient from whom we have previously obtained an OCT scan the pathological sample and the OCT scan would be obtained at a different stage of the disease and not reflect the same pathology. It would also not be possible to obtain exactly the same section on postmortem histology and on OCT scan for direct comparison.

OCT angiography (OCTA) would ideally show perfusion deficits in the different retinal plexuses to compare localisation with hyper-reflective dots on OCT b-scans. However, OCTA cannot prove the changes within the vessels and in the retinal tissues such as hyper-reflective zones corresponding to PAMM in the inner retinal layers.

Also, paediatric OCT angiography (OCTA) is not available and OCTA requires stable fixation for at least 27 seconds for scanning a similar area on the OCT B-scans. ${ }^{36}$ The hand-held OCT data are collected in very difficult circumstances on the malaria ward on comatose children. Examiners need to constantly adapt the position of the OCT probe to the position of the patient's body and eyes flexibly to obtain OCT b-scans. One b-scan takes only 0.025 seconds. It is not possible to fix the OCT probe for a longer duration during this examination to obtain OCTA. Examiners need to constantly adapt the position of the OCT probe to the position of the patient's position, as they make involuntary body and eye movements. Although there are limitations in our study, we have used the best dataset in an endemic setting and the best available knowledge comparing the pathology to conventional fluorescein angiography (see Fig. 1, 2 and supplementary figure S3) and post-mortem pathology from other 
patients carefully to reach the current hypothesis. Although our interpretation of data is to some extend speculative our paper presents important new findings which could serve as biomarker for cerebral malaria.

\section{Conclusion}

Our study using non-invasive OCT imaging of the retina has generated new insights into the pathophysiology of MR and CM. The novel finding of hyper-reflective abnormalities in capillaries and vessels most likely corresponds to sequestered pRBCs within these vessels. Rapid disappearance of this hyper-reflectivity indicates that OCT may allow to monitor the effect of treatment on pRBCs sequestration and clearance in vivo. OCT clearly differentiated retinal whitening (HRA affecting the inner retinal layers), from cotton wool spots (affecting the RNFL). HRAs are more common than visible retinal whitening from ischemia. Follow-up data indicated that HRAs lead to retinal atrophy, suggesting a mechanism for cerebral atrophy and high rates of cognitive and developmental impairment after CM.

OCT changes are specific to CM patients with MR, and allow differentiation between malarial and non-malarial coma. We have shown that $\mathrm{HH}-\mathrm{OCT}$ is a fast, non-invasive method to image neural tissue, especially for young children, its microvasculature and how they are affected by CM in humans, both acutely and in the long-term. It can provide insights into pathophysiology but also potentially for better clinical care.

\section{Declarations}

\section{Competing interests}

The author(s) declare no competing interests.

\section{Author contributions statement}

Dr. Zhanhan Tu - Literature search, study design, data collection, conceived and designed and performed data analysis, data interpretation, figures and tables and manuscript preparation.

Dr. Jack Gormley - Literature search, data collection in Malawi and manuscript preparation.

Dr. Viral Sheth - Literature search, data collection, data analysis and proofreading.

Dr. Karl B. Seydel - Patient recruitment, study design, data interpretation and manuscript preparation.

Professor. Terrie Taylor - Patient recruitment, study design, data interpretation and manuscript preparation.

Mr. Nicholas Beare - Help with interpretation of results and proofreading.

Dr. Valentina Barrera - Provided histological images and interpretation.

Dr. Frank A Proudlock - Designed data analysis and proofreading.

Mr. Chatonda Manda - Data collection.

Professor Simon Harding - Study design and setting up a collaboration with Malawi, critical review of the manuscript. 
Professor Irene Gottlob - Literature search, study design, funding of the project, setting up a collaboration with Malawi, designed data analysis, data interpretation and manuscript preparation.

\section{References}

1 Seydel, K. B. et al. Brain swelling and death in children with cerebral malaria. N Engl J Med 372, 1126-1137, doi:10.1056/NEJMoa1400116 (2015).

2 Postels, D. G., Chimalizeni, Y. F., Mallewa, M., Boivin, M. J. \& Seydel, K. B. Pediatric cerebral malaria: a scourge of Africa. Future Neurology 8, 67-85, doi:10.2217/fnl.12.84 (2013).

3 WHO. World Malaria Report 2018. (2018).

4 Langfitt, J. T. et al. Neurodevelopmental Impairments 1 Year After Cerebral Malaria. Pediatrics 143, e20181026, doi:10.1542/peds.2018-1026 (2019).

5 Taylor, T. E. et al. Differentiating the pathologies of cerebral malaria by postmortem parasite counts. Nat Med 10, 143-145, doi:10.1038/nm986 (2004).

6 MacPherson, G. G., Warrell, M. J., White, N. J., Looareesuwan, S. \& Warrell, D. A. Human cerebral malaria A quantitative ultrastructural analysis of parasitized erythrocyte sequestration. Am J Pathol 119, 385 (1985 Jun).

7 White, N. et al. Pathophysiological and prognostic significance of cerebrospinal-fluid lactate in cerebral malaria. The Lancet 325, 776-778, doi:https://doi.org/10.1016/S0140-6736(85)91445-X (1985).

8 Turner, G. D. H. et al. Systemic endothelial activation occurs in both mild and severe malaria. Correlating dermal microvascular endothelial cell phenotype and soluble cell adhesion molecules with disease severity. Am J Pathol 152, 1477-1487 (1998 Jun).

9 Elhassan, I. M. et al. Evidence of Endothelial Inflammation, T Cell Activation, and T Cell Reallocation in Uncomplicated Plasmodium Falciparum Malaria. The American Journal of Tropical Medicine and Hygiene 51, 372379, doi:doi:https://doi.org/10.4269/ajtmh.1994.51.372 (1994).

10 Brown et al. Evidence of blood-brain barrier dysfunction in human cerebral malaria. Neuropathology and Applied Neurobiology 25, 331-340, doi:doi:10.1046/j.1365-2990.1999.00188.x (1999).

11 Newton, C. R. J. C. et al. Intracranial hypertension in Africans with cerebral malaria. Arch Dis Child 76, 219-226 (1997 Mar 1).

12 Clark, I. A., Rockett, R. A. \& Cowden, W. B. TNF in cerebral malaria. QJM 86, 217-218 (1993 Mar 1).

13 Clark, I. A., Cowden, W. B. \& Rockett, K. A. Nitric oxide in cerebral malaria. J Infect Dis 171, 1068-1069 (1995).

14 Clemens, R. et al. Activation of the coagulation cascade in severe falciparum malaria through the intrinsic pathway. Br J Haematol 87, 100-105 (1994).

15 WHO. in Tropical Medicine \& International Health Vol. 19 7-131 (2014). 
16 Barrera, V. et al. Neurovascular sequestration in paediatric P. falciparum malaria is visible clinically in the retina. Elife 7, doi:10.7554/eLife.32208 (2018).

17 White, V. A., Lewallen, S., Beare, N. A., Molyneux, M. E. \& Taylor, T. E. Retinal pathology of pediatric cerebral malaria in Malawi. PLoS One 4, e4317, doi:10.1371/journal.pone.0004317 (2009).

18 Beare, N. A. et al. Prognostic significance and course of retinopathy in children with severe malaria. Arch Ophthalmol 122, 1141-1147, doi:10.1001/archopht.122.8.1141 (2004).

19 Maldonado, R. S. et al. Optimizing hand-held spectral domain optical coherence tomography imaging for neonates, infants, and children. Invest Ophthalmol Vis Sci 51, 2678-2685, doi:10.1167/iovs.09-4403 (2010).

20 Molyneux, M. E., Taylor, T. E., Wirima, J. J. \& Borgsteinj, A. Clinical Features and Prognostic Indicators in Paediatric Cerebral Malaria: A Study of 131 Comatose Malawian Children. QJM: An International Journal of Medicine 71, 441-459, doi:10.1093/oxfordjournals.qjmed.a068338 (1989).

21 MacCormick, I. J. et al. Grading fluorescein angiograms in malarial retinopathy. Malar J 14, 367, doi:10.1186/s12936-015-0897-7 (2015).

22 Hero, M. et al. Photographic and angiographic characterization of the retina of kenyan children with severe malaria. Archives of Ophthalmology 115, 997-1003, doi:10.1001/archopht.1997.01100160167005 (1997).

23 Sorsby, A., Sheridan, M., Leary, G. A. \& Benjamin, B. Vision, visual acuity, and ocular refraction of young men: findings in a sample of 1,033 subjects. British medical journal 1, 1394-1398, doi:10.1136/bmj.1.5183.1394 (1960).

24 Rahimy, E., Kuehlewein, L., Sadda, S. R. \& Sarraf, D. Paracentral Acute Middle Maculopathy: What We Knew Then and What We Know Now. Retina (Philadelphia, Pa.) 35, 1921-1930, doi:10.1097/iae.0000000000000785 (2015).

25 Barrera, V. et al. Severity of retinopathy parallels the degree of parasite sequestration in the eyes and brains of malawian children with fatal cerebral malaria. J Infect Dis 211, 1977-1986, doi:10.1093/infdis/jiu592 (2015).

26 Harding, S. P. et al. Classifying and grading retinal signs in severe malaria. Trop Doct 36 Suppl 1, 1-13, doi:10.1258/004947506776315781 (2006).

27 Paquet-Durand, F. et al. A retinal model of cerebral malaria. Sci Rep 9, 3470, doi:10.1038/s41598-019-39143-z (2019).

28 Lawrence, C. \& Olson, J. A. Birefringent hemozoin identifies malaria. Am J Clin Pathol 86, 360-363 (1986).

29 Matthias Bolz et al. Optical Coherence Tomographic Hyperreflective Foci: A Morphologic Sign of Lipid Extravasation in DiabeticMacular Edema. Ophthalmology 116, 914-920 (2009).

30 Beare, N. A., Harding, S. P., Taylor, T. E., Lewallen, S. \& Molyneux, M. E. Perfusion abnormalities in children with cerebral malaria and malarial retinopathy. J Infect Dis 199, 263-271, doi:10.1086/595735 (2009).

31 Lochhead, J. et al. The effects of hypoxia on the ERG in paediatric cerebral malaria. Eye (Lond) 24, 259-264, doi:10.1038/eye.2009.162 (2010). 
32 Sarraf, D. et al. Paracentral acute middle maculopathy: a new variant of acute macular neuroretinopathy associated with retinal capillary ischemia. JAMA Ophthalmol 131, 1275-1287, doi:10.1001/jamaophthalmol.2013.4056 (2013).

33 Casalino, G., Williams, M., McAvoy, C., Bandello, F. \& Chakravarthy, U. Optical coherence tomography angiography in paracentral acute middle maculopathy secondary to central retinal vein occlusion. Eye 30, 888, doi:10.1038/eye.2016.57 (2016).

34 Bhavsar, K. V. et al. Acute macular neuroretinopathy: A comprehensive review of the literature. Survey of Ophthalmology 61, 538-565, doi:https://doi.org/10.1016/j.survophthal.2016.03.003 (2016).

35 McLeod, D. Why cotton wool spots should not be regarded as retinal nerve fibre layer infarcts. Br J Ophthalmol 89, 229-237, doi:10.1136/bjo.2004.058347 (2005).

36 Talisa E de Carlo, Andre Romano, Nadia K Waheed \& Duker1, J. S. A review of optical coherence tomography angiography (OCTA). International Journal of Retina and Vitreous 1, doi:10.1186/s40942-015-0005-8 (2015).

\section{Figures}



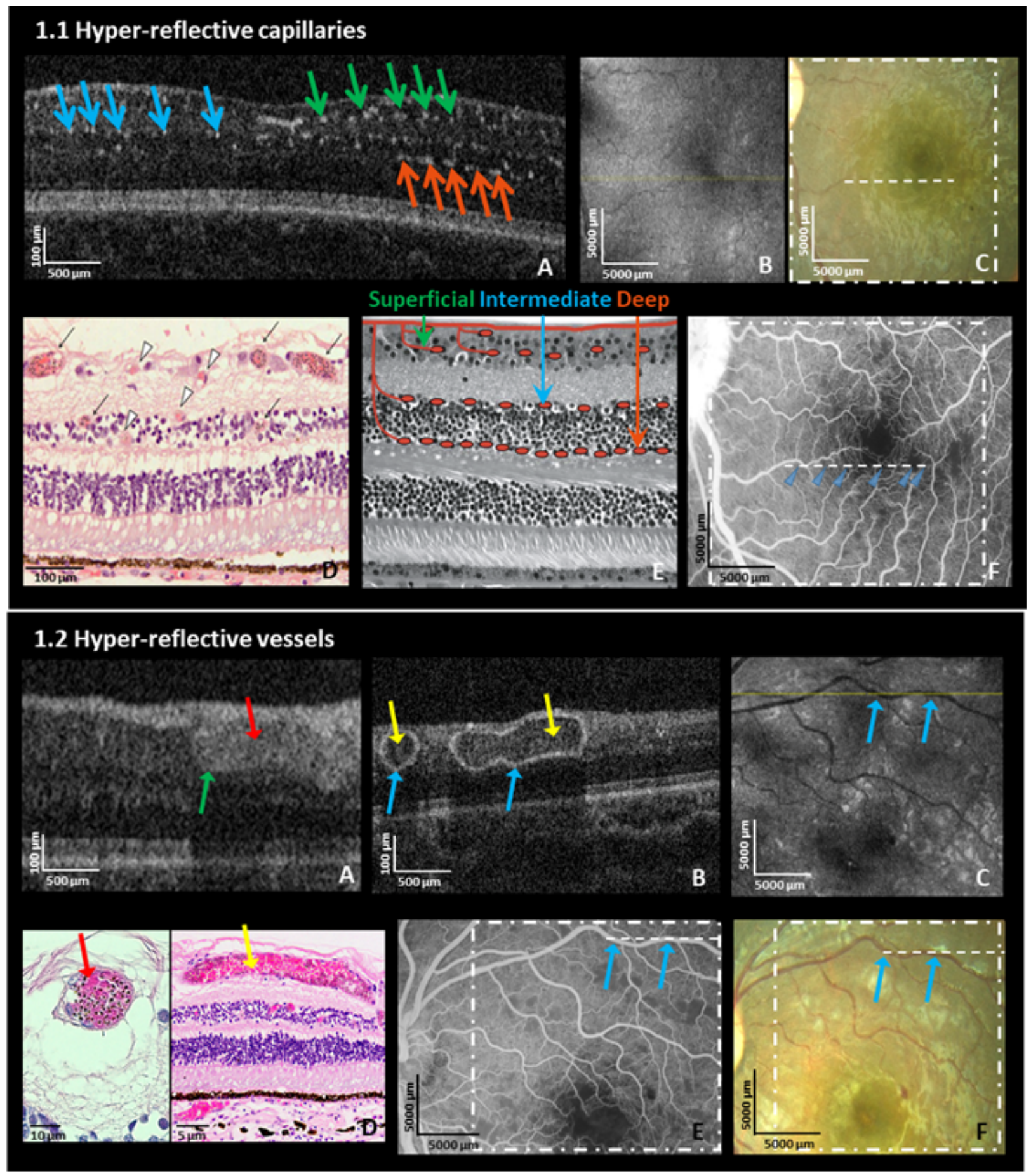

\section{Figure 1}

Hyper-reflective capillaries and vessels 1.1. Left eye of a seven-month old male CM patient on admission.A. HH-OCT B-scan showing hyper-reflective dots corresponding to locations of capillaries (green, blue and orange arrows: superficial, intermediate and deep capillary plexus); B. En-face OCT displays no visible change; (yellow line: Iocation of the OCT B-scan in image A). C. Fundus photo; dashed line and square correspond to A and B); D. Representative retinal cross-section histology from a different fatal patient showing capillaries and venules affected by parasite sequestration (black arrows: intense sequestration; arrowheads: low or no sequestration); Reprinted with permission25;E. Schematic representation of superficial,intermediate and deep capillary plexus collocated to capillaries filled with pRBCs on OCT in A. Reprinted with permission24; F. Fundus fluorescein angiography (FA) shows perfusion deficits of capillaries (blue arrows) corresponding to parasitized vessels on OCT in the same 
location of 1.1.A. 1.2. Left eye of a 42-month old male CM patient on admission.A. Hyper-reflective vessel (blue arrow) corresponding to localization of vessel on fundus photo and FA (not shown) with hyper-reflective lumen (red arrow) ; and B. Hyper-reflective vessels (blue arrows) with hypo-reflective lumina (yellow arrows) on OCT B-scans; C.En-face OCT (yellow line: location of OCT B-scan in B, blue arrows: vessels shown in B; D. Representative retinal cross-section histology showing pRBCs in venules from a different fatal patient (red arrow shows vessel with pRBCs filling the lumen; yellow arrow shows a vessel where pRBCscytoadhere to the vessel wall with red blood cells without parasites in the center of the vessel (similar to hypo-reflective lumina of blood vessels in 1.2.B); E. Fundus fluorescein angiography and F. Fundus photo corresponding to OCT in 1.2.B (blue arrows corresponding to vessels in image B; dashed line and square correspond to B and C).
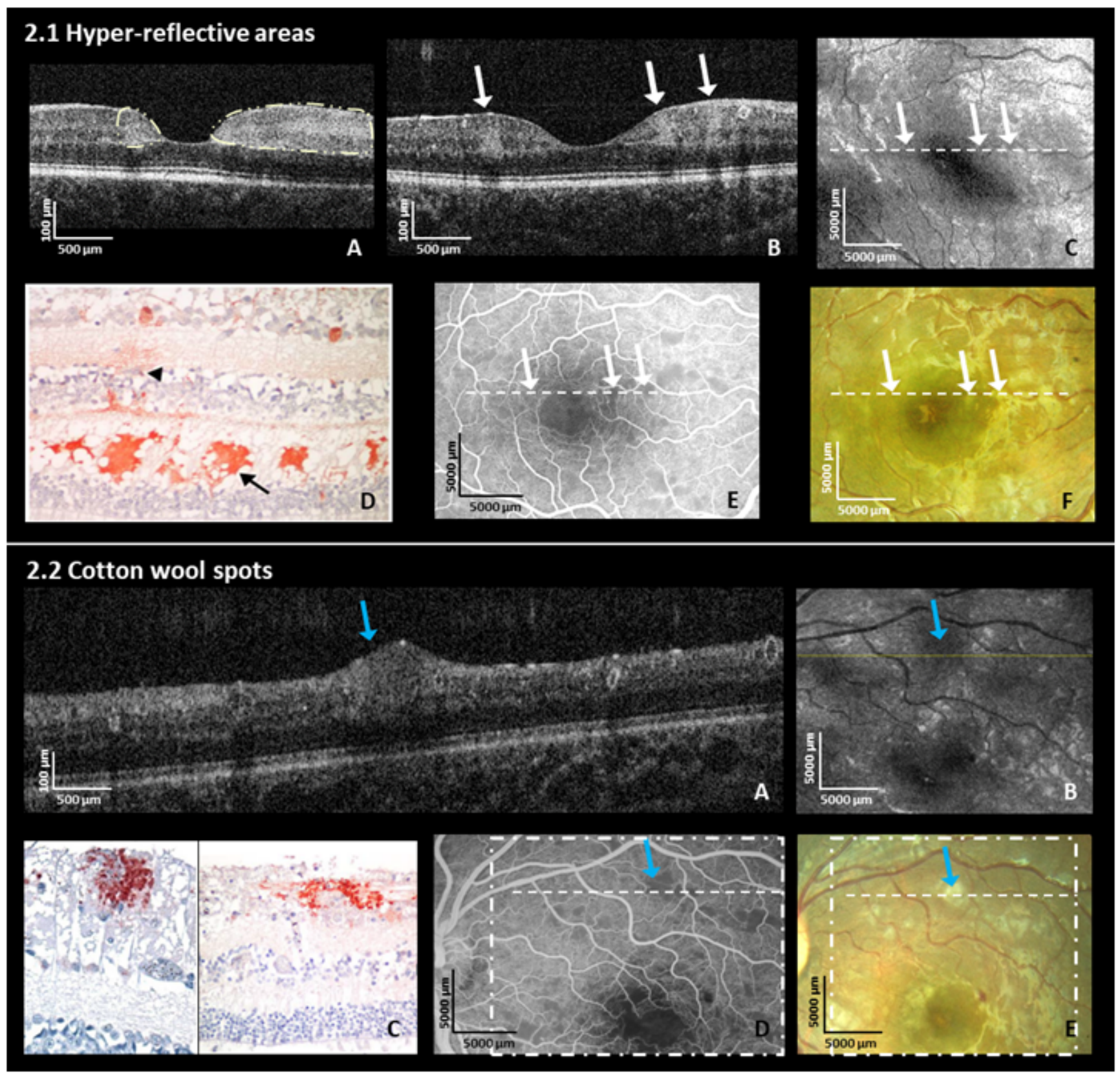

\section{Figure 2}

Hyper-reflective areas and cotton wool spots (Retinal Whitening) 2.1.A and B shows multiple hyper-reflective areas of various sizes (white arrows) located in inner nuclear layer level, the outer plexiform layer and outer nuclear layer on OCT B-scans in right eye of a 22-month old male CM patients and in left eye of a 84-month old female CM 
patient at admission respectively; C. En-face OCT of patient in B; white arrows correspond to location of white arrows in image B (hyper-reflective areas); The hyper-reflective areas in OCT B-scan (B) show darkening on the enface image (C) in this patient (white arrows); D. Representative histology of immuno-histochemical staining for fibrinogen surrounding a small vessel (black arrowhead) mainly located in OPL (black arrow) from the literature (Reprinted with permission) 17E. Fundus fluorescein angiography showing hypo-perfusion of capillaries and F. fundus photo same eye as OCT in B; white arrows and dashed lines show parafoveal whitening which are corresponding to the same fundus locations in B,C,E and F. 2.2.A. Cotton wool spot (blue arrow) in the left eye at admission on OCT B-scan in the left eye of a 42-months old male CM patient at admission; B.En-face OCT with darkening in area of cotton wool spot (blue arrow); fine yellow line: location of the B-scan in image A; blue arrow corresponds to blue arrow in image $A$; $C$. Immunohistochemical staining for b-APP in retinal nerve fiber layer (RNFL) from different CM patients. Reprinted with permission17D. Fundus fluorescein angiography showing grey area masking underlying capillaries (blue arrow) and E. fundus photo from patient's left eye corresponding to OCT showing white cotton wool spot (blue arrow) in 2.2.A. The dashed square and the dashed line correspond to en-face OCT in B and B-scan in A. Blue arrows show location of cotton wool spot. Blue arrows correspond to the same fundus locations in $A, B, D$ and $E$. 


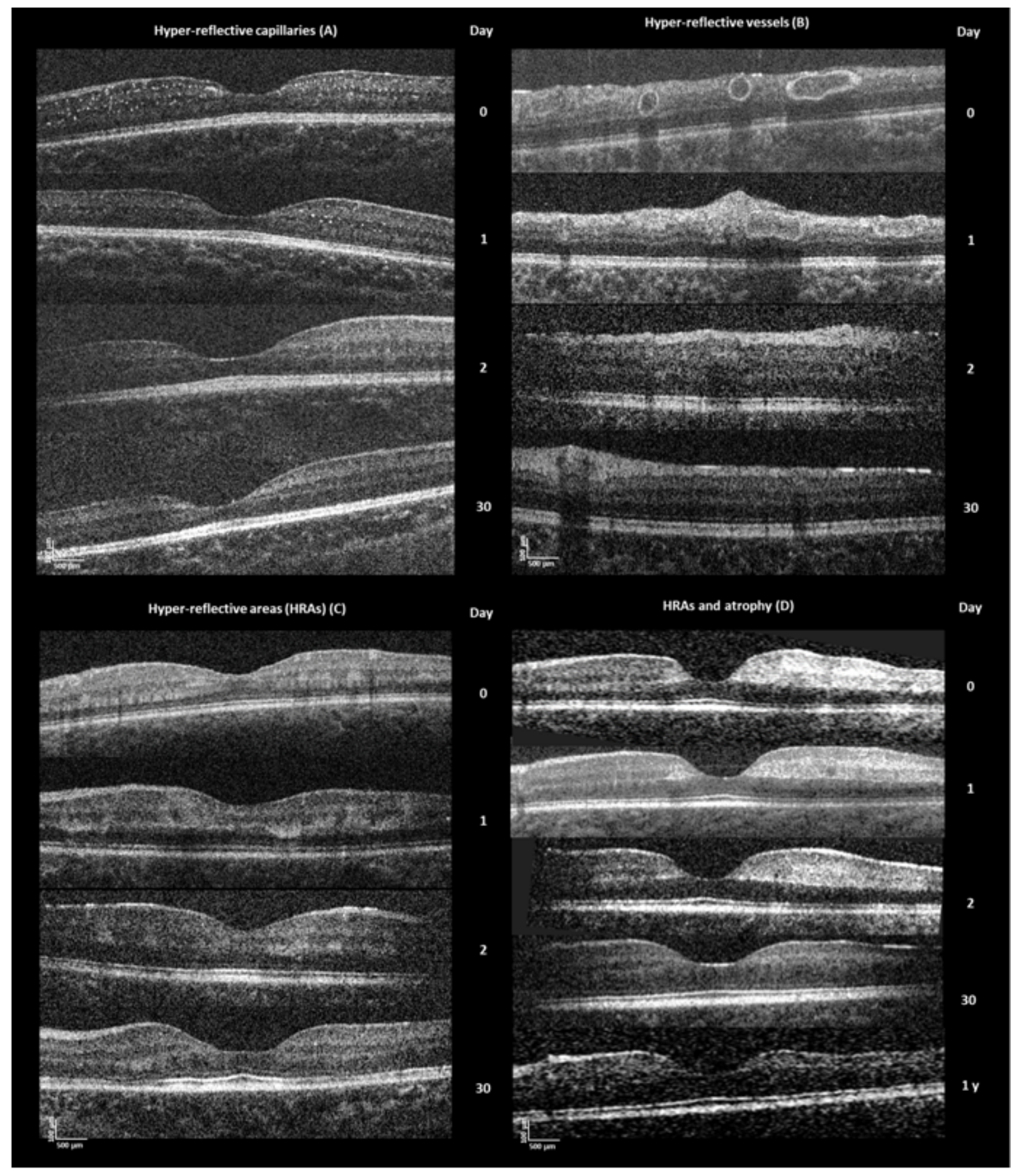

\section{Figure 3}

Longitudinal changes in the retina A.Hyper-reflective capillaries: OCT of a seven-month-old male CM patient at admission, one day, two days and 30 days. The number of hyper-reflective capillaries diminished rapidly one day after treatment initiation; hyper-reflective capillaries were almost not detectable on OCT after two days. B. Hyperreflective vessels: OCT of 42-month-old male CM patient at admission, one day, two days and 30 days after start of treatment. The abnormal hyper-reflective vessels also diminished rapidly within 24 hours. C. Hyper-reflective areas: OCT of 45-month-old female CM patient at admission, one day, two days and 30 days after start of treatment. The hyper-reflective areas remained visible, but were smaller and less intense at one-month follow-up. Retinal atrophy was just evident at this time point. D. Parafoveal hyper-reflective areas and atrophy: OCT of 22-month-old male CM patient at admission, one day, two days, 30 days and one year. He had relatively large and dense hyper-reflective 
areas at admission (particularly to the temporal side of the fovea (right)). There is corresponding retinal atrophy at one-year follow-up with marked thinning compared to the left of the fovea.

\section{A. Comparison of retinal layers between 1-month follow-up in $\mathrm{CM}$ patients and healthy controls}

Inner retinal layers (whole CM group)

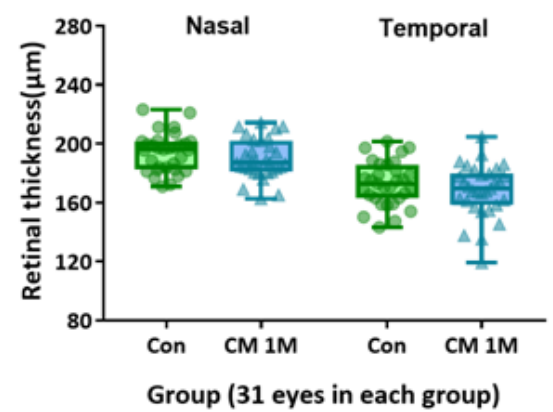

Inner retinal layers (CM with HRAs)

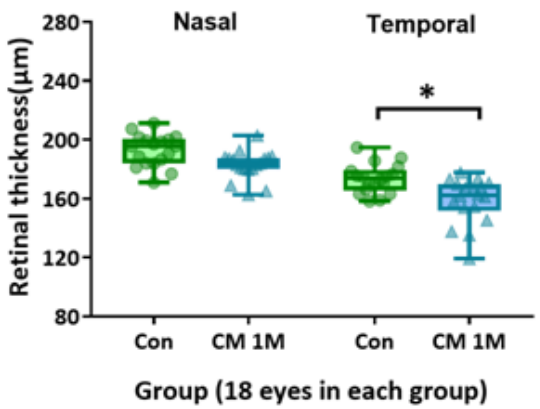

Inner retinal layers (CM without HRAs)

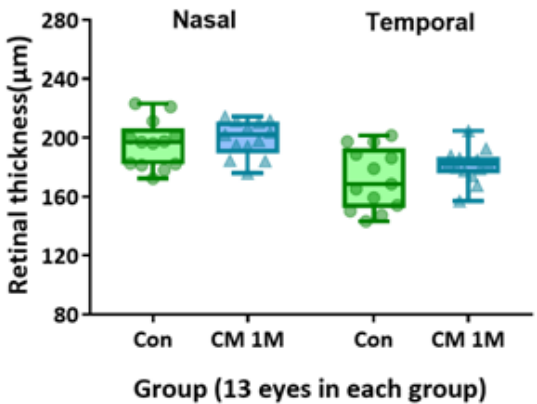

B. Comparison of retinal layers between 1-year follow-up in CM patients and healthy controls

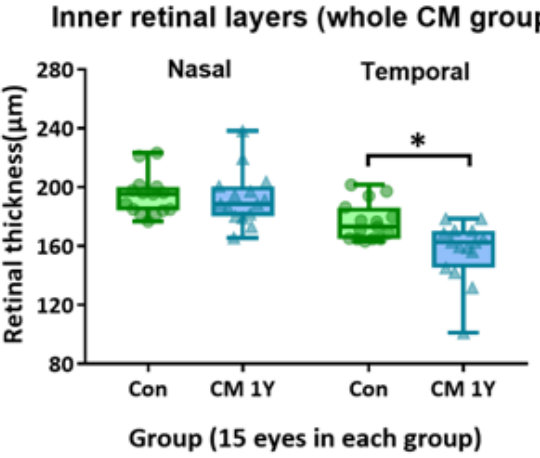
Inner retinal layers (CM with HRAs)

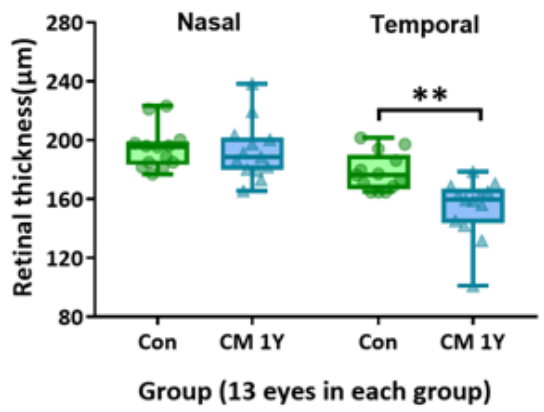

Figure 4

Comparisons of thicknesses of retinal layers between follow-up of CM patients with HRAs and healthy controls A: Comparison of thickness of inner retinal layers between controls and CM patients at one-month follow-up. B: Comparisons of thicknesses of inner retinal layers between controls and CM patients at one-year follow-up. Error bars in boxplot are the ranges of data. $*$ : $p<0.05$ and **: $p<0.01$ show significant different between two groups. Control groups are in green and patient groups in blue (Con= controls; $\mathrm{CM}=$ cerebral malaria; $1 \mathrm{M}=$ one-month follow-up; $1 Y=$ one-year follow-up)

\section{Supplementary Files}

This is a list of supplementary files associated with this preprint. Click to download.

- SupplementaryDatacleanversion.pdf 